\title{
Applying the Holistic Approach to the Physical Sciences and Engineering Systems
}

\author{
Joseph Z. Ben-Asher \\ Aerospace Engineering, Technion, Israel Institute of Technology, Haifa, Israel
}

Received 2012-02-26, Revised 2012-07-13; Accepted 2013-08-20

\begin{abstract}
The holistic approach is applied to several examples from the field of physics, engineering and systems engineering and its benefits are demonstrated. In all examples a holistic picture is used and studied while ignoring the various details of the discussed problems. Optimal control and optimal estimation approachs are used in the engineering examples. The results show that the holistic approach provides a deeper insight into the main phenomena while requiring much fewer computational resources Moreover, in all cases the details may significantly be changed yet leading to similar observed phenomena. Whenever applicable, the holistic approach is highly recommended.
\end{abstract}

Keywords: Conservation Laws, Flexible Spacecraft, Optimal Control, Extended Kalman Filter (EKF), Resources Allocation, Data-Fusion, Holistic Properties

\section{INTRODUCTION}

A definition to systems engineering contributed by Simon Ramo is as follows:

"Systems engineering is a discipline that concentrates on the design and application of the whole (system) as distinct from the parts. It involves looking at a problem with its entirety, taking into account all the facts and all the variables and relating the social to the technical aspects" (Haskins, 2007).

The holistic approach had been recognized in the physical sciences and in the various fields of engineering for many years, long before the birth of systems engineering. Although in all other fields it seems to be consequential-in systems engineering it is an essential property (hence it becomes part of its definition). One may say that the essence of systems engineering is its being holistic. The top-down approach also stems from viewing the system as a whole (Blanchard and Fabrycky, 1998).

What exactly is the meaning of the holistic approach regarding systems? One way to express it is that "the whole is not just the sum of its parts" (attributed to Aristotle) in the sense that emergent properties of a complex system cannot be reconstructed from its simplest components. This again is taken from the natural sciences as eloquently expressed by Anderson (1972).

"The ability to reduce everything to simple fundamental laws does not imply the ability to start from those laws and reconstruct the universe. The constructionist hypothesis breaks down when confronted with the twin difficulties of scale and complexity. At each level of complexity entirely new properties appear. Psychology is not applied biology, nor is biology applied chemistry. We can now see that the whole becomes not merely more, but very different from the sum of its parts"(Anderson, 1972).

Another way of interpreting the holistic approach is somewhat less drastic and associates it to the human cognitive capacities rather than to the objective world. In this sense we do not claim that emergent properties cannot be reconstructed from lower level properties but rather that it is much simpler for the human mind to understand a system by overlooking the various details and by concentrating on the holistic properties themselves. It also deepens the understanding in the sense of discriminating between the essential and the accidental. 
This study adopts the softer version of the two interpretations when dealing with systems engineering issues. Note that no claim is made regarding the other view (i.e., the view that emergent properties are not possessed by any one of the parts) with respect to natural sciences in general and this problem is beyond the scope of this study (e.g., see the discussion in (Hofstadter, 1999).

The purpose of the study is to demonstrate the holistic approach and its great benefits by several examples from physics, engineering and finally from system engineering. For the latter a systems engineering case study will be given for a multi sensor system and data fusion.

\section{CONSERVATION LAWS IN PHYSICS AND ENGINEERING}

We begin this section by a very simple puzzle example: Suppose that you have two identical cups: one with a certain amount of liquid A, say coffee and the other with identical amount of liquid B, say milk. Assume equal specific density for A and B. Take one tea spoon of coffee and pour it into the milk cup. Then, after well stirring, take one tea spoon from the mixture and return it to the coffee cup. Repeat this process three times (same order of cups-do not switch).

Question: By the end of the process which cup has more foreign liquid Cup A (originally with coffee), or Cup B (originally with milk)?

There are two ways to approach the problem. The first (what most people follow-especially engineers) is to calculate at each step the various amounts in the cups using all the mixture rules. The other one is holistic and, as a matter of fact, is process independent. At the end of the process consider the two cups as in Fig. 1. Clearly the total liquids are of the same height $\mathrm{h}$ (equal amounts of total liquid; equal densities) because of the principle of mass conservation. Now if Cup A has $x$ of the new liquid there must be (h-x) of the original one in this cup. Hence the amount that has been removed to Cup B must be $x$ as well. One can appreciate the simplicity and clarity of the holistic approach. Not only it renders the solution simple, but it also provides an important insight into the results, enabling a generalization of the problem in hand. One is losing the picture when dwelling into the details even when the correct answer is obtained.

The second problem is taken from mechanics (Feynman et al., 1963). Consider a mass of $1 \mathrm{lb}$ sitting in equilibrium on the inclined plane with a pulley which has a weight $\mathrm{W}$ on the other hand. Assume, for simplicity, that the inclined plane is a 3-4-5 Pythagorean triangle (Fig. 2).

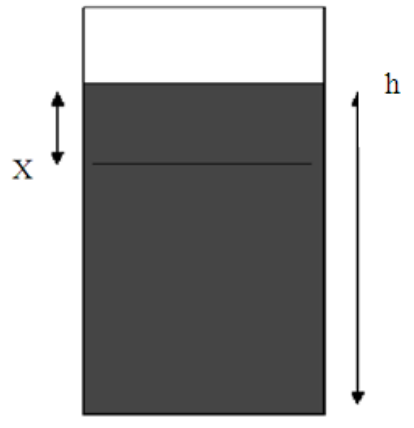

(A)

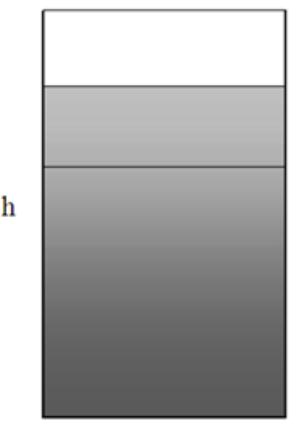

(B)
Fig. 1. The coffee and milk puzzle

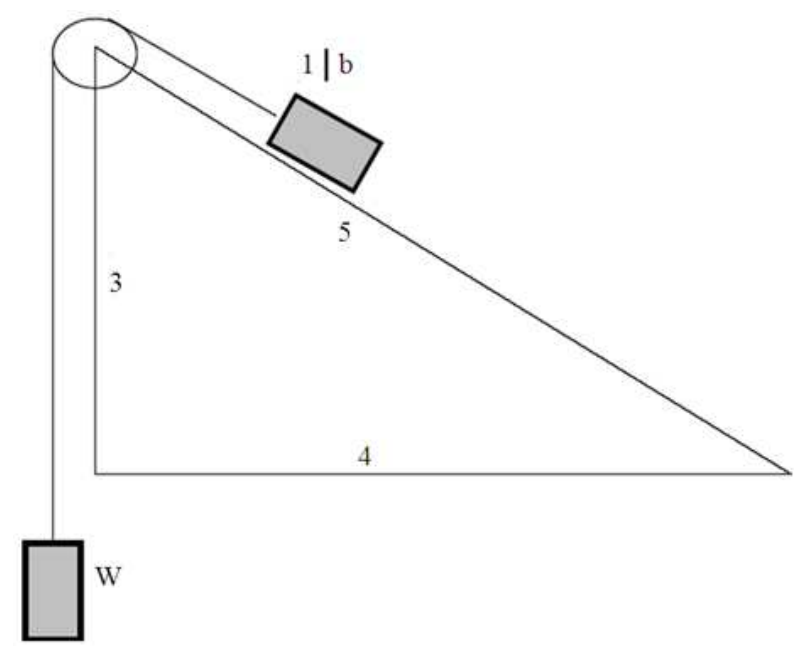

Fig. 2. Mechanical equilibrium

Question: What should be the value of W?

Here again one can solve the problem using equilibrium of forces in all directions. The pulley tension is one of the applied forces; the inclined plane reactions and gravity are the other applying forces. Conservation of energy however provides a much simpler approach. Due to equilibrium we can move the system up and down without doing any amount of work. Consider the situation where the $1 \mathrm{lb}$ mass is at the bottom and another situation where this mass is at the top. Clearly we have moved the mass by 5 feet and at the same time, the mass $\mathrm{W}$ losses 5 feet of heightthus $5 \mathrm{~W} \mathrm{lb}$ - $\mathrm{ft}$ is the corresponding loss in its potential energy. Our $1 \mathrm{lb}$ mass gained $3 \mathrm{lb}$-feet of potential energy and from the conservation of energy we must have $\mathrm{W}=3 / 5 \mathrm{lb}$.

Our third example is a vertical gyro (see a detailed description in (Merchav, 1996). A vertical gyro is a device used to measure the pitch $(\theta)$ and roll 
angles $(\varphi)$ of a vehicle (usually-an aircraft). This quite complicated device is depicted in Fig. 3. The spin of the heavy mass is rotating in the vertical direction. The potentiometer reading of inner gimbal provides the pitch angle measurement and that of the outer gimbal-the roll measurement.

In order to keep the spin in its vertical position, an erection mechanism is employed by which two small pendulums, attached to the inner gimbal, sense the spin deviation (caused by drift) and close circuits which activate two small electrical motors $\left(T_{1}\right.$ and $\left.T_{2}\right)$ to correct the vertical drift at a certain precession rate say PR. When the mother vehicle is accelerating this erection mechanism is cut-off to avoid aligning the spin axis with the total instantaneous acceleration vector.

Evidently the whole gyro is quite a complex electro-mechanical system. In a specific project it was required to evaluate PR by a laboratory test. Since acceleration values higher than $1 \mathrm{~g}$ (gravity) were needed it was proposed to use a centrifugal table as shown in Fig. 4. It was supposed that the spin-under the operation of the erection circuits-would align itself with the total acceleration namely the vector sum of gravity and $\Omega^{2}$ R.

The test was performed in the laboratory but the observed phenomenon was a perfect alignment with the vertical (or very nearly to it). The next trial was to cut-off the erection circuits and to re-run the test (spinning the table by $\Omega$ ): The result was virtually the same-a perfect (or nearly perfect) alignment with the vertical direction.

Question: What was going on?

Once again there could be two ways to address this question. The first one is to write down the dynamic equations (non-linear differential equations), to analyze them (especially is steady state) and from this analysis to get the explanation. The second one is holistic and immediate. Notice that for the spin to align itself with the instantaneous acceleration vector it must change its direction continuously due to the table rotation $\Omega$. But conversation of angular momentum prohibits it-unless some appropriate external moments are applied which is clearly not the case here (albeit some small moments are obtained from the erection motors).

So we should not have expected the alignment with the acceleration vector. But the question remains why (in both cases of erection on and erection off) the almost perfect alignment with the vertical? To answer simply that it aligns itself with the table rotation vector is not enough-Earth rotates the Sun while keeping its spin axis in a fixed direction. The answer is again quite simple. Due to friction (and maybe mass-imbalance) we get the effect of gyro compassing. In a nut-shell gyro-compassing is obtained whenever there are external moments which disappear at a certain equilibrium point. When the spin axis of the table and the gyro spins axis are aligned, there is no relative movement of the gimbals, the moments then vanish and we have equilibrium.

Notice again how the holistic approach provides answers and insights for some very complicated problems.

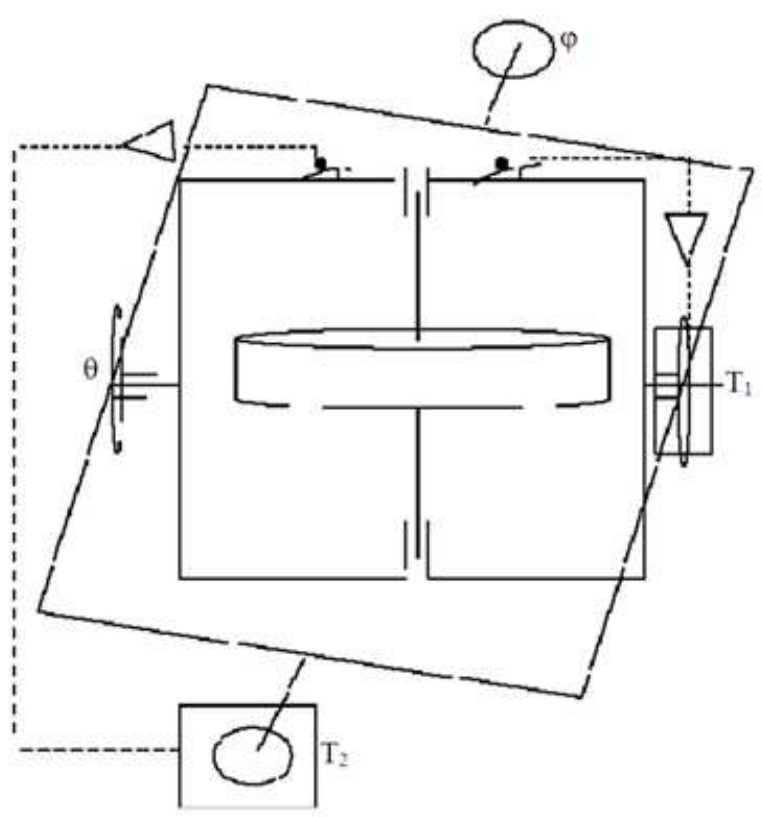

Fig. 3. Vertical gyroscope

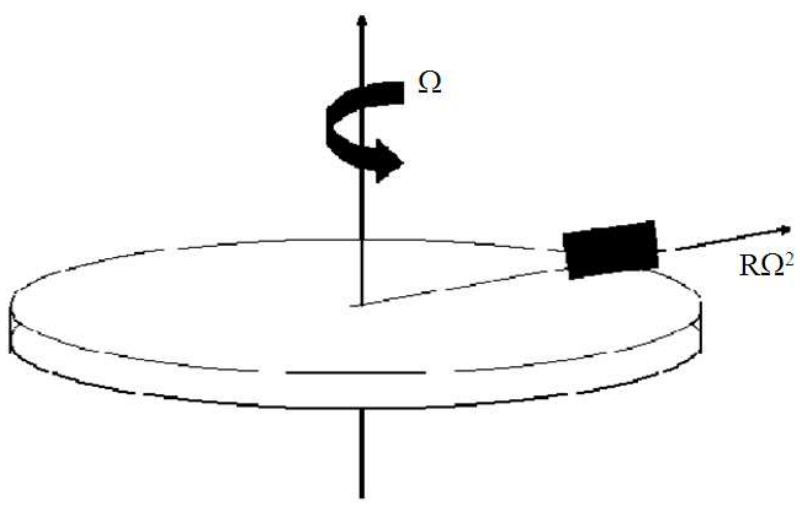

Fig. 4. Centrifugal table 


\section{SYMMETRY IN PHYSICS AND ENGINEERING}

The last section dealt with holistic approach based on conservation laws (mass, energy and momentum). Another road for holistic interpretation and problem solving in sciences and Engineering is via symmetric properties of space and time. The following case study-taken from the author's Ph.D. dissertation (BenAsher, 1988)-exploits both symmetries.

Over the past two decades, time optimal attitude maneuvers for flexible spacecraft have become a topic of great interest. In particular, a system consisting of a rigid hub controlled by a single actuator, with one or more elastic appendages attached to the hub, was studied by several researchers who investigated the properties of minimum-time rest-to-rest rotational maneuvers (as well as other maneuvers). Minimum maneuvers have a bang-bang solution-the control is alternating between maximal and minimal value (maximal negative). This system represents, under certain assumptions, a satellite with a rigid hub and flexible solar panels modeled as Euler-Bernoulli beams (Fig. 5). Minimum time rotational maneuvers of satellites have important scientific and strategic applications. The dynamic equations can be shown to have the following form:

$\ddot{\eta}(\mathrm{t})+\Omega^{2} \eta(\mathrm{t})=\mathrm{Gu}(\mathrm{t})$

$\Omega^{2}=\left[\begin{array}{llll}0 & & & \\ & \omega_{1}^{2} & & \\ & & \ddots & \\ & & & \omega_{\mathrm{n}}^{2}\end{array}\right]$

Here $\eta=\left[\eta_{0}, \eta_{1}, \eta_{2}, . . \eta_{3}\right]^{\mathrm{T}}$ is a vector of generalized coordinates and $\omega_{\mathrm{i}}$ is the $\mathrm{i}^{\text {th }}$ natural frequency. Notice that the first equation corresponds to the rigid mode motion $\left(\omega_{0}=0\right)$ whereas the rest are equations for the first $\mathrm{n}$ flexible modes. The scalar $\mathrm{u}(\mathrm{t})$ is an external moment exerted on the rigid hub.

We pose the following optimal control problem: Find the time optimal control $\mathrm{u}(\mathrm{t}) ; 0 \leq \mathrm{t} \leq \mathrm{T}$ and the corresponding state trajectories $\bar{\eta}(t)$ that the system is driven from the initial conditions:

$$
\begin{aligned}
& \bar{\eta}(0)=[-\Theta, 0 . .0] \\
& \dot{\bar{\eta}}(0)=[0,0 . .0]
\end{aligned}
$$

To the final conditions at the origin:

$$
\begin{gathered}
\bar{\eta}(T)=[0,0 . .0] \\
\dot{\bar{\eta}}(T)=[0,0 . .0]
\end{gathered}
$$

These boundary conditions express a rest-to-rest maneuver whereby the spacecraft is rotated by a positive angle $\Theta$.

The problem was solved numerically for various degrees of freedom (Fig. 6-8). The solution is bang-bang with an increasing number of switching points (as function of the degree of freedom).

It was observed that a certain and very useful symmetric property is always valid, namely:

$$
\overline{\mathrm{u}}(\mathrm{t})=-\overline{\mathrm{u}}(\mathrm{T}-\mathrm{t})
$$

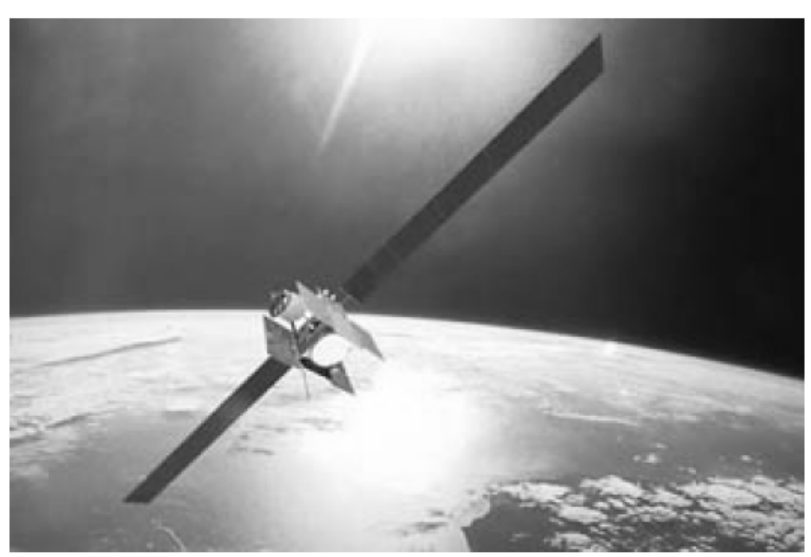

Fig. 5. Flexible spacecraft

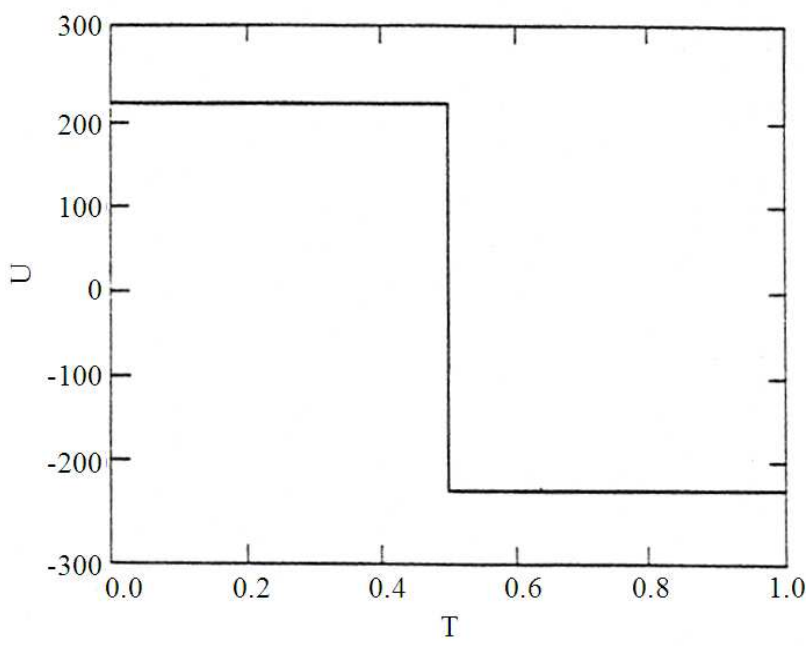

Fig. 6. Rigid body 


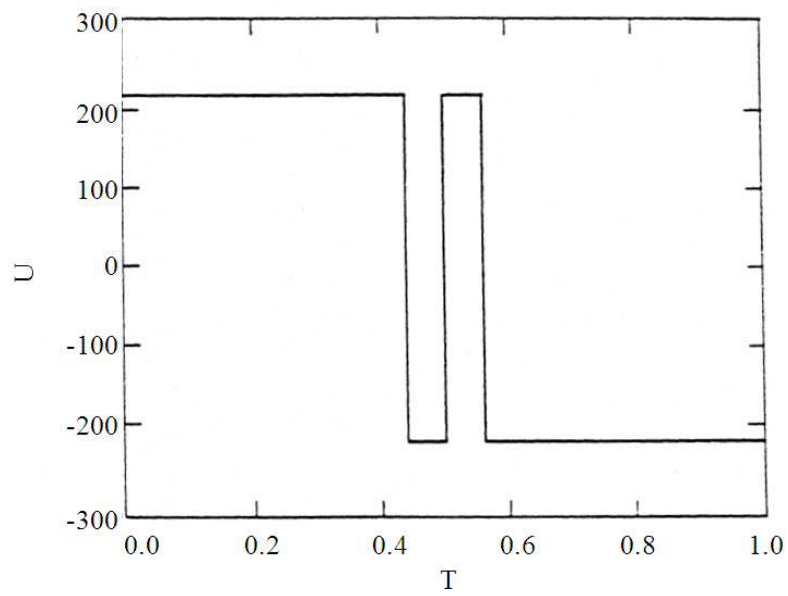

Fig. 7. One flexible mode

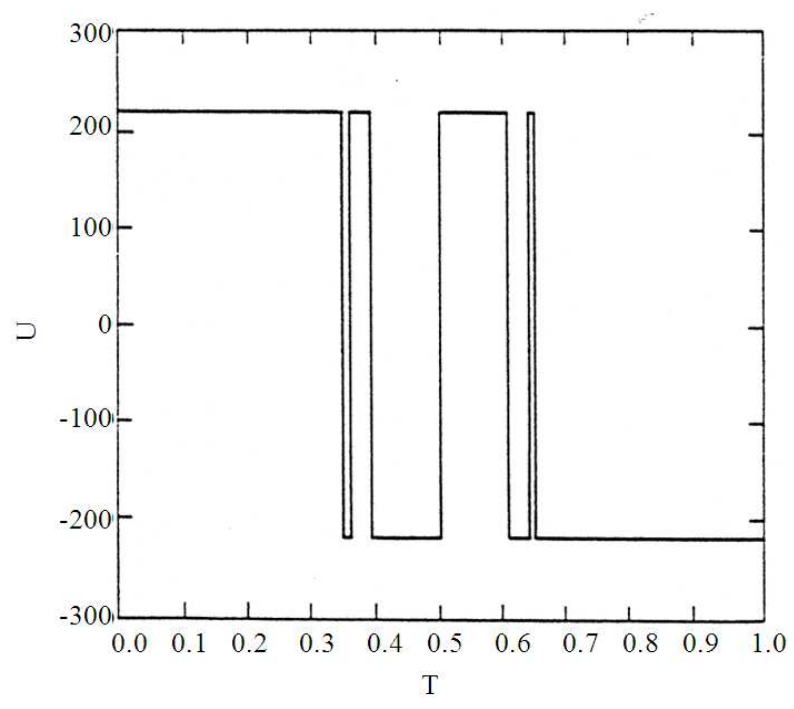

Fig. 8. Two flexible modes

Moreover, if we change the cost from minimum time to minimum control effort i.e.:

$$
J=\int_{0}^{T} u^{2}(t) d t
$$

Then Equation 4 still holds (albeit the bang-bang structure is no longer the solution).

Question: why?

To answer this curious question one can use the detailed formulation of the Minimum Principle and workout through the resulting two-point boundaryvalue problem (Singh et al., 1989). The computational effort is high and is dependent on the particular cost function. A different holistic approach was proposed in (Ben-Asher et al., 1992). This approach exploits the observation that this problem has both time and space symmetries.

First notice that if we rotate the spacecraft in the opposite direction, e.g., from:

$$
\begin{gathered}
\bar{\eta}(0)=[0,0 . .0] \\
\dot{\bar{\eta}}(0)=[0,0 . .0] \\
\text { To: } \\
\bar{\eta}(\mathrm{T})=[-\Theta, 0 . .0] \\
\dot{\bar{\eta}}(\mathrm{T})=[0,0 . .0]
\end{gathered}
$$

We must obtain the same minimum time $\mathrm{T}$ by exerting the opposite control function:

$$
\overline{\bar{\mu}}(\mathrm{t})=-\bar{\mu}(\mathrm{t})
$$

Moreover, because Equation 1 is symmetric with time, we may reformulate our original problem using the reversed time:

$$
\tau \equiv \mathrm{T}-\mathrm{t}
$$

The dynamics equation then becomes:

$$
\frac{\mathrm{d}^{2} \eta(\tau)}{\mathrm{d} \tau^{2}}+\Omega^{2} \eta(\tau)=\mathrm{Gu}(\tau)
$$

The boundary conditions are in reversed time Equation 5 and 6 (instead of Equation 2 and 3), hence, Equation 7 is our optimal control function:

$$
\overline{\overline{\mathrm{u}}}(\tau(\mathrm{t})=-\overline{\mathrm{u}}(\tau(\mathrm{t}))
$$

Recalling that since we are still dealing with the original problem the value of the control at $t$ must be $\mathrm{u}(\mathrm{t})$ (the control has the same value at time $\mathrm{t}$, regardless of the way it has been obtained).

Hence:

$\overline{\overline{\mathrm{u}}}(\tau(\mathrm{t}))=\overline{\mathrm{u}}(\mathrm{t})$

From Equation 8, 10 and 11 we arrive at: 
$-\overline{\mathrm{u}}(\mathrm{T}-\mathrm{t})=\overline{\mathrm{u}}(\mathrm{t})$

Notice that the observation in Equation 12 is for both costs. Notice also that had damping been included in Equation 1 and 9 is no longer valid as time symmetry is broken.

\section{A CASE STUDY IN SYSTEMS ENGINEERING USING THE HOLISTIC APPROACH}

The common thread of the above case studies is the observation that working out the problem detailseven though may eventually lead to the problem solution-is a tedious process which can become redundant when the holistic view is adopted. Moreover, the latter provides a deeper insight into the physical phenomena rendering its use indispensible. The next case study demonstrates the same trends in a systems engineering problem regarding data fusion in ballistic missile and rocket defense systems.

This kind of defense systems uses phased array radars for various missions such as search, tracking, discrimination (i.e., target identification in cluster). (Naveh and Lorber, 2001). Missions "compete" over the same finite stockpile of sensor resources and have to be performed within certain time intervals. Mission performance level depends on the amount of sensor resources allocated to it and therefore can be optimized by specific allocations.

An interim problem of the general resource optimization is the radar tracking beam allocation problem. Classically, the objective of the sensor allocation process in tracking has been to minimize the uncertainty in the tracking estimation error of all relevant targets, using a given amount of radar resources. This problem has been addressed by (Israeli et al., 2009) where open-loop optimal strategies were obtained using direct optimization. The essentially bang-zero-bang structure of the solutions was investigated by extensive numerical solutions and the main features of the optimal strategies were characterized. Introducing ElectroOptical Sensor (EOS) to the defense system calls for data fusion of the optical and radar sensors. The angular accuracy of the optical sensor is superior to the radar's thus data fusion obtained by a common filter using both measurements should have superior performance relative to the radar in stand alone operation. A study similar to (Israeli et al., 2009) of finding optimal tracking policies in order to obtain a required accuracy with minimum amount of resources has been recently conducted.

One of the questions involved in this kind of research is where should be the best location for the EOS.

For a stand-alone EOS (i.e., target tracking is based on angular measurements as provided by this sensor-bearing only estimation) the answer had been known before. Figure 9 presents the time history for the obtained accuracy (in terms of the variance of the radial uncertainty as computed by an Extended Kalman Filter -EKF) for two representative cases (identical ballistic missile trajectory). The accuracy is given using certain distance units (DU) and as a function of time measured by certain Time Units (TU). Case A is for the EOS located on the ballistic plane at the impact point whereas Case B is with the EOS location shifted by 250 DU off the ballistic plane (transversal). Case B provides much better results and so locating the EOS off the ballistic place is superior in this case.

Under data fusion a combined Extended Kalman Filter (EKF) was investigated for tracking the target (Bar-Shalom et al., 2001; Bryson and Ho, 1975). The covariance is propagated as given in Appendix A. Fixing the radar resources to its minimum values $\left(\mathrm{u}_{1}\right)$ required for continuous tracking-we search for the minimum required EOS total resources $\left(\mathrm{u}_{2}\right)$ to achieve a required accuracy at a certain time.

Several locations were considered as depicted in Fig. 10. The optimal tracking resources measured in time units of occupation needed for a given accuracy are given in Table 1.

As shown the effect of the EOS location is highly significant. Consider first the case $\mathrm{Y}=0$ where the two sensors (radar and EOS) are located in the ballistic plane. Decreasing the range from EOS to target along the same direction improves the data-fusion predicted accuracy thus decreases the EOS load. This phenomenon is well understood as the position errors grow with range. When we locate the EOS near the ballistic plane at $\mathrm{Y}=100 \mathrm{DU}$ this is still the case. However this phenomenon is reversed when we are way off the ballistic plane (last row). Moreover, the behavior along the columns is also surprising. Based on the EOS stand-alone performance of we might expect less resources when moving away from the ballistic plane, but this is clearly not the case here. For example the last column tells us that the resources are three folded when we move 250 DU away from the origin (co-location of sensors) in the $\mathrm{Y}$ direction and it gets even worse when we move forward. 


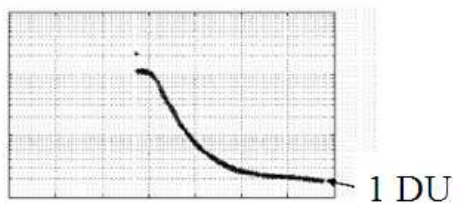

(A)

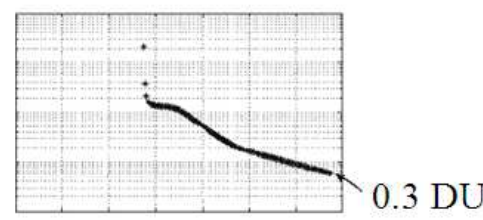

(B)

Fig. 9. EOS Stand-Alone Prediction Accuracy (A) EOS location [0,0] (B) EOS location [0,250 DU]

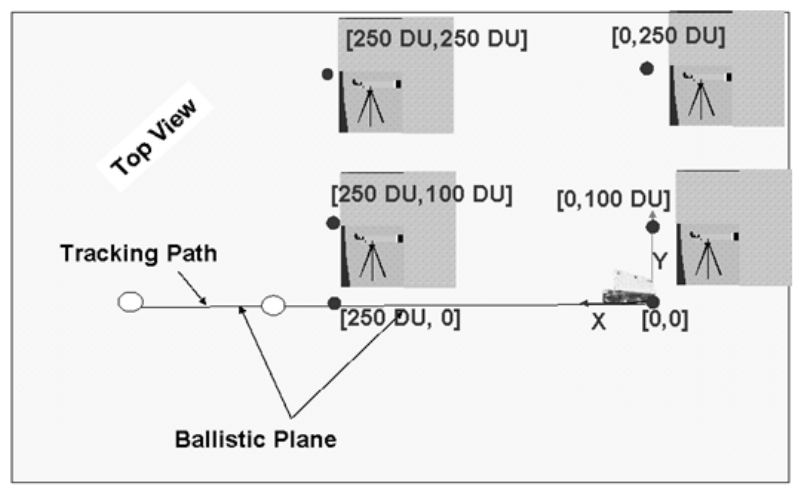

Fig. 10. EOS Locations

Table 1. EOS resources (occupation time) Vs. EOS location

\begin{tabular}{lll}
\hline & $\mathrm{X}=250 \mathrm{DU}$ & $\mathrm{X}=0$ \\
\hline $\mathrm{Y}=0$ & $2 \mathrm{TU}$ & $4 \mathrm{TU}$ \\
$\mathrm{Y}=100 \mathrm{DU}$ & $2 \mathrm{TU}$ & $5 \mathrm{TU}$ \\
$\mathrm{Y}=250 \mathrm{DU}$ & $25 \mathrm{TU}$ & $12 \mathrm{TU}$ \\
\hline
\end{tabular}

Question: why?

Here again there could be two ways to approach the question. The first is a tedious study of all the details involved in the data fusion process. We may investigate the observability measures of the combined EKF and the influence of the location on its properties. We may go into an even lower level and ask the question of what the value of a single EOS measurement ( $1 \mathrm{TU})$ is and how it depends on the EOS location.

The other approach is holistic. We investigate the question of what is the best EOS location for a system working as a whole. Recall that the EOS provides angular measurement to the target with very high accuracy. The radar (as all tracking radars) is extremely accurate in range measurement but has relatively poor angular measurements. Thus, the best location for the EOS must be on the line-of-sight between the radar and the target where it optimally complements the radar at its weakest point. In our example it should be on the ballistic plane. Moreover, if the EOS is in the transverse point-such as $\mathrm{X}=250$
$\mathrm{DU}, \mathrm{Y}=250$ DU-then the joint performance is the poorest. The azimuth measurements of the EOS at this location are in fact redundant due to the excellent range measurements of the radar and all the benefits we get stem from the EOS elevation measurementshence the large amount of resources needed for the required performance.

We observe here an interesting property that the optimal setting of a subsystem if different when in standalone position and in joint operation with another subsystem. In particular, the property of "being in the best location" as part of the whole is very different than the same property in a stand-alone operation.

\section{CONCLUSION}

Several case studies from physics, engineering and systems engineering have been presented. The main lessons learned is that working out the problem details may be a tedious process that can become superfluous if the holistic view is adopted. The deeper insight into the physical phenomena obtained by the holistic view renders its use indispensible. Moreover in many cases the details may significantly be changed yet leaving the main phenomena intact.

\section{REFERENCES}

Anderson, P.W., 1972. More is different. Science, 177: 393-396. DOI: $10.1142 / 9789812385123$ others01

Bar-Shalom, Y., X.R. Li and T. Kirubarajan, 2001. Estimation with Applications to Tracking and Navigation. 1st Edn., John Wiely and Sons, New York, ISBN-10: 047141655X, pp: 584.

Ben-asher, J., J.A. Burns and E.M. Cliff, 1992. Timeoptimal slewing of flexible spacecraft. J. Guidance Control Dynamics, 15: 360-367. DOI: $10.2514 / 3.20844$

Ben-Asher, J.Z., 1988. Time Optimal Slewing of Flexible Spacecraft. Ph.D. Thesis, Virginia Polytechnic Institute and State University.

Blanchard, B.S. and W.J. Fabricky, 1998. Systems Engineering and Analysis. 3rd Edn., Prentice Hall, Upper Saddle River, N.J., ISBN-10: 0131350471, pp: 738. 
Bryson, A.E. Jr. and Y.C. Ho, 1975. Applied Optimal Control: Optimization, Estimation and Control. 1st Edn., Taylor and Francis, ISBN-10: 0891162283, pp: 496.

Feynman, R.P., R.B. Leighton, M.L. Sands and M.A. Gottlieb, 1963. The Feynman Lectures on Physics. 2nd Edn., Addison-Wesley, ISBN-10: 0805390499, pp: 384.

Haskins, C., 2007. INCOSE Systems Engineering Handbook: A Guide for Sytem Life Cycle Processes and Activities. 1st Edn., INCOSE, Seattle, Wash.

Hofstadter, D.R., 1999. Gödel, Escher, Bach: An Eternal Golden Braid. 20th Edn., Basic Books, New York, ISBN-10: 0465026567, pp: 824.

Israeli, N., J.Z. Ben-Asher and D. Cohen, 2009. Radar tracking optimization for ballistic target scenarios. Proceedings of the AIAA Guidance, Navigation and Control Conference, Aug. 10-13, Chicago, Illinois. DOI: $10.2514 / 6.2009-6222$

Merchav, S., 1996. Aerospace Sensor Systems and Applications. 1st Edn., Springer, New York, ISBN10: 0387946055 , pp: 454.

Naveh, B.Z. and A. Lorber, 2001. Theater Ballistic Missile Defense. 1st Edn., American Institute of Aeronautics and Astronautics, Reston, VA., ISBN10: 1563473852 , pp: 397.

Singh, G., P.T. Kabamba and N.H. McClamroch, 1989. Planar, time-optimal, rest-to-rest slewing maneuvers of flexible spacecraft. J. Guidance, 12: 71-81.

\section{APPENDIX A:}

In this appendix we will briefly present the Extended Kalman Filter used for data fusion in the fourth section (based on (Bar-Shalom et al., 2001; Bryson and Ho, 1975).

The point mass model of a ballistic target can be written as:

$$
\begin{aligned}
& \frac{d x}{d t}=f_{1}\left(x, y, h, v, \gamma, y_{D}\right)=v \cdot \cos (\gamma) \\
& \frac{d y}{d t}=f_{2}\left(x, y, h, v, \gamma, y_{D}\right)=y_{D} \\
& \frac{d h}{d t}=f_{3}\left(x, y, h, v, \gamma, y_{D}\right)=v \cdot \sin (\gamma) \\
& \frac{d v}{d t}=f_{4}\left(x, y, h, v, \gamma, y_{D}\right)= \\
& -\frac{1}{2} \rho(h) v^{2} \beta^{\prime}-g \cdot \sin (\gamma)+w_{1} ; \quad \beta^{\prime}=\frac{C_{D} S}{m} \\
& \frac{d \gamma}{d t}=f_{5}\left(x, y, h, v, \gamma, y_{D}\right)=-\cos (y) \cdot \frac{g}{v}+\frac{w_{2}}{v} \\
& \frac{d y_{D}}{d t}=f_{6}\left(x, y, h, v, \gamma, y_{D}\right)=0
\end{aligned}
$$

Where:

$$
\begin{array}{ll}
\mathrm{x} & =\text { Down range } \\
\mathrm{y} & =\text { Cross range } \\
\mathrm{y}_{\mathrm{D}} & =\text { Cross range rate } \\
\mathrm{h} & =\text { Altitude } \\
\mathrm{v} & =\text { Velocity } \\
\gamma & =\text { Dive angle } \\
\mathrm{m} & =\text { Mass } \\
\mathrm{C}_{\mathrm{D}} & =\text { Drag coefficient } \\
\mathrm{S} & =\text { Reference area } \\
\rho & =\text { Density } \\
\mathrm{W}_{1}, \mathrm{~W}_{2} & =\text { Perturbation forces }
\end{array}
$$

The radar measures range, elevation and azimuth $\{\Psi$ $\Theta \mathrm{R}\}$ to the target and the EOS measures elevation and azimuth to the target $\left\{\Psi_{1} \Theta_{1}\right\}$. Denote $\mathrm{g}$ as the measurment vector $\left[\Psi \Theta \mathrm{R} \Psi_{1} \Theta_{1}\right]$. The following is the propagation of covariance matrix $\mathrm{P}$ :

$$
\begin{aligned}
& \mathrm{P}=\mathrm{M}-\mathrm{MC}^{\mathrm{T}}\left[\mathrm{CMC}^{\mathrm{T}}+\mathrm{R}\right]^{-1} \mathrm{CM} \\
& \mathrm{M}=\mathrm{APA}^{\mathrm{T}}+\mathrm{LQL}^{\mathrm{T}} \\
& \mathrm{A}=\frac{\partial \mathrm{f}_{\mathrm{i}}}{\partial \mathrm{x}_{\mathrm{j}}}+\delta_{\mathrm{ij}} ; \mathrm{C}=\frac{\partial \mathrm{g}_{\mathrm{i}}}{\partial \mathrm{x}_{\mathrm{j}}} \\
& \mathrm{L}=\left[\begin{array}{cccccc}
0 & 0 & 1 & 0 & 0 & 0 \\
0 & 0 & 0 & 1 / \mathrm{v} & 0 & 0
\end{array}\right]^{\mathrm{T}} ; \mathrm{Q}=\left[\begin{array}{cc}
\sigma_{\mathrm{w}_{1}}^{2} & 0 \\
0 & \sigma_{\mathrm{w}_{2}}^{2}
\end{array}\right] \\
& \mathrm{R}_{1}=\left[\begin{array}{ccc}
\sigma_{\psi}^{2} & 0 & 0 \\
0 & \sigma_{\theta}^{2} & 0 \\
0 & 0 & \sigma_{\mathrm{r}}^{2}
\end{array}\right] ; \mathrm{R}_{2}=\left[\begin{array}{cc}
\sigma_{\psi_{1}}^{2} & 0 \\
0 & \sigma_{\theta_{1}}^{2}
\end{array}\right] ; \mathrm{R}=\left[\begin{array}{cc}
\frac{1}{\mathrm{u}_{1}} \mathrm{R}_{1} & 0 \\
0 & \frac{1}{\mathrm{u}_{2}} \mathrm{R}_{2}
\end{array}\right] \\
& 0<\mathrm{u}_{1} \leq 1 \quad 0<\mathrm{u}_{2} \leq 1
\end{aligned}
$$

Note that here we are approximating the optimal allocations by using $\mathrm{u}_{1}$-radar partial allocation-and $\mathrm{u}_{2}$-EOS partial allocation-that are being kept constant for $1 \mathrm{TU}$. 